\title{
Dealing with the Uncertainty of Having Incomplete Sources of Geo-Information in Spatial Planning
}

\author{
L. A. E. Vullings • C. A. Blok • C. G. A. M. Wessels • \\ J. D. Bulens
}

Received: 4 January 2011 / Accepted: 24 February 2012 /

Published online: 30 March 2012

C The Author(s) 2012. This article is published with open access at Springerlink.com

\begin{abstract}
The Dutch spatial planning legal act of 2008 was aimed at improving efficiency and effectiveness in the development, evaluation and monitoring of spatial planning policy (Ministry of VROM, 2006a). One of the main effects of this legal act was the widespread availability and use of digital spatial plans (Ministry of VROM $2006 \mathrm{a}, \mathrm{b})$. This reform led to the expectation that all digital spatial plans would be exchangeable and comparable. In practice, this exchange and comparison required carrying out complex procedures due to uncertainty caused by differences in the scope of spatial plans as well as their intended use. Furthermore the uncertainty resulted in a lack of confidence in spatial plans by policymakers and supporting GIS staff. Our overarching research question was: how can uncertainty caused by incomplete geo-information sources be dealt with? We proposed two techniques-fuzzy logic and visualisation - for policy makers to deal with uncertainty resulting from incomplete geo-information sources in spatial planning at the regional and national planning levels. We used two case studies in the Netherlands to illustrate the results of applying these techniques. The fuzzy set theory provides extra information by converting the discrete borders of continuous objects into fuzzy borders that improve the resemblance to the real object and thus make it more realistic. As shown in the second case study, visualisation also improves the degree of realism and thus provides additional information. Both case studies showed that providing additional information reduces the uncertainty felt by policymakers.
\end{abstract}

Keywords Uncertainty · Spatial planning · Geo-information · Fuzzy set theory · Visualisation

\footnotetext{
L. A. E. Vullings $(\bowtie) \cdot J$. D. Bulens

Alterra, Wageningen University and Research Centre, P.O. Box 47, 6700, AA Wageningen, The Netherlands

e-mail: Wies.vullings@wur.nl

C. A. Blok

Geo-Information Processing Department, Faculty of Geo-Information Science and Earth Observation (ITC) of the University of Twente, P.O.Box 217, 7500 AE Enschede, The Netherlands

C. G. A. M. Wessels

Nexpri V.O.F., Ondiep Zuidzijde 6, 3551 BW Utrecht, The Netherlands
} 


\section{Introduction}

Recent changes in spatial planning legislation in the Netherlands have resulted in a lack of confidence among planning officials in planning procedures. This was primarily due to various sources of uncertainty in the procedures and a lack of experience in dealing with this uncertainty.

The Dutch spatial planning legal act of 2008 was aimed at improving efficiency and effectiveness in the development, evaluation and monitoring of spatial planning policy (Ministry of VROM 2006a). One of the main effects of this legal act was the widespread availability and use of digital spatial plans (Ministry of VROM 2006a; Ministry of VROM 2006b) for consultation by the public and/or governmental bodies at various stages throughout a spatial planning process (Ministry of VROM 2010).

A spatial plan is usually an envisioning of a political choice with a status that can vary. A plan is composed of planning objects. These are spatial features with specific properties, including a reference to a linked description in a legal text. Digitising these spatial plans resulted in changes in the procedures related to the gathering of geo-information sources for supporting a choice (usually a political one), as well the procedures that produce spatial plans as part of a spatial planning process. Together, these developments led to a reform of the Dutch spatial planning process.

This reform led to the expectation that all digital spatial plans would be exchangeable and comparable: exchangeable in terms of interoperable between authorities of different levels (local, regional and national) and comparable in terms of content. In practice, this exchange and comparison has been limited to the intended scope of the digital plans. Due to the differences in the scope of spatial plans as well as their intended use, complex procedures were required. Some plans only gave global indications of a desired future land use, while others were very detailed and precise - for example specifying building permits and restrictions on citizens at a local level. Additional complexity in the procedures was due to the different spatial scales, with associated levels of detail and temporal scales, of the geo-information sources used for the creation of digital spatial plans. Moreover, typical aspects of data quality such as errors in the datasets, semantic ambiguity, unknown lineage and unknown temporal accuracy led to uncertainty.

These differences in intended use, level of detail, temporal scale and data quality resulted in a lack of confidence in spatial plans by policymakers and supporting GIS staff. This was caused by possible sources of uncertainty in the procedures and data, as well as the overall quality of digital plans (Vullings and de Vries 2007; Vullings et al. 2009; Wessels et al. 2008). An overview of possible sources of uncertainty and how to deal with uncertainty within a spatial planning process is crucially important for supporting this on-going reform.

Our overarching research question was: how can uncertainty caused by incomplete geo-information sources be dealt with? We proposed two techniques-fuzzy logic and visualisation - for policy makers to deal with uncertainty resulting from incomplete geo-information sources in spatial planning at the regional and national planning levels. We used two case studies in the Netherlands to illustrate the results of applying these techniques.

The remainder of the paper is organised as follows. Section 2 elaborates on the conceptualisation of uncertainty with respect to spatial planning. Section 3 describes the first case study on regional spatial planning: finding an optimal location for greenhouses 
within the city triangle of Apeldoorn, Deventer and Zutphen in the Netherlands. Section 4 describes the second case study at the national spatial planning level; it focused on the visualisation of uncertainty in national spatial plans. Finally, Section 5 concludes the paper with a discussion on the usefulness of the proposed techniques for dealing with uncertainty in supporting the Dutch Spatial Planning legal act.

\section{The Definition of Uncertainty in Spatial Planning}

Many definitions exist for uncertainty related to geo-information sources. Leyk et al. (2005) provided a very useful overview of the research issues and definitions. They used the definition proposed by Fisher (2003), in which uncertainty is defined as the doubt about the information that is recorded at some location. Zhang and Goodchild (2002) defined uncertainty as a measure of the difference between the data and the meaning attached to the data by a current user. According to Fisher (1999) and Atkinson and Foody (2002), uncertainty can also be seen as an umbrella term consisting of concepts such as result of error, ambiguity, vagueness or lack of information.

In this paper we have adopted the definition of uncertainty as the acknowledgement that one does not know the context of a planning object exactly because of incomplete sources of geo-information. Hereby we assume that it is not the planning object that is uncertain, but instead, we are the ones who are uncertain about this object. In other words, we do not know enough about a planning object in order to understand its impact properly.

Incompleteness is defined here as a lack of relevant geo-information that has resulted in only a partial description of a planning object (Bejaoui 2009). This incompleteness can lead to logical contradictions during the generation of digital spatial plans (Worboys and Duckham 2004). There are several approaches proposed in the literature for handling incomplete data, including logistic regression (Williams et al. 2005), Bayesian networks (Beal and Ghahramani 2003), and Hilbert space (Gao et al. 2010). Williams et al. (2005) main contribution was the derivation of a missingdata logistic regression classification algorithm. By making two mild assumptions, the algorithm solves the incomplete-data problem in a principled manner, avoiding imputation heuristics. Beal and Ghahramani (2003) presented an efficient procedure for estimating the marginal likelihood of probabilistic models with latent variables or incomplete data. Gao et al. (2010) proposed a method widely used in image restoration to apply to the reconstruction of irregular spaced seismic data by interpolating missing traces. They conclude that their method is method is fit for interpolating reconstruction of linear and quasi-linear events.

In this paper we propose two easy-to-use and easy-to-understand methods that can be used by policymakers. The following two case studies describe ways of dealing with uncertainty caused by incompleteness in sources of geoinformation for spatial planning by using two different techniques to provide more information about the planning object. We have chosen to work with the fuzzy set theory in the first case study, because it deals with continuous objects with no discrete borders. According to Shi (2010) and Comber et al. (2005), fuzzy set theory is suitable for vague objects. In the first case study, fuzzy set theory was 
used to facilitate the use of continuous phenomena that are commonly represented by discrete objects, because fuzzy object more closely resemble continuous phenomena. In the second case study we looked at visualisation techniques that aim at explicitly communicating incompleteness in data to create awareness among users of a spatial plan. Users are more confident about their decisions when uncertainties related to spatial planning objects are visualised on digital plans (Edwards and Nelson 2001).

\section{Case Study 1: 'Finding an Optimal Location for Greenhouses within the City Triangle of Apeldoorn, Deventer and Zutphen in the Netherlands'}

The aim of this case study was to describe how the fuzzy logic technique can be used to handle the uncertainty about the light pollution and noise emission that would result from the allocation of greenhouses in the city triangle. The uncertainty was due to a lack of relevant geo-information about the potential noise emission within the statutory buffer zones surrounding nature conservation areas.

\section{Background}

In the Netherlands, space is a valuable asset. Often the allocations of new functions have to take place within areas already claimed entirely by other functions, leaving no space for other developments. This case study is an example of such an allocation problem that was ascertained by the Dutch Ministry of Agriculture and the Dutch Cadastre when searching for an optimal location to cluster the greenhouses in the city triangle of Apeldoorn, Deventer and Zutphen in the Netherlands (Vullings et al. 2009; Wessels et al. 2008). The region around these three cities had already been claimed by agrarian functions, as approved in the rural plans of the municipalities, and by nature conservation areas (Natura2000 sites and Dutch ecological main structure) and their $3 \mathrm{~km}$ protection zones (crisp buffer zones). Within the buffer zones certain activities, such as agrarian functions, are excluded due to the potential impact of ammonia emissions on the nature areas.

These existing claims considerably hinder any spatial development for a large concentration of greenhouses. Legally, the allocation of greenhouses is bounded by the $3 \mathrm{~km}$ protection zones of the existing nature conservation areas based on the generic ammonia emission effect. However, the environmental impact of greenhouses is not caused by ammonia emission, but is related to light pollution (photosynthetic lighting at night) from the greenhouses and noise emission caused by increasing transportation of products to and from the greenhouse operations. Both light pollution and noise emission have less impact on the existing nature conservation areas than ammonia emission.

Therefore, we proposed a fuzzy logic approach to define the protection zones. Instead of having crisp objects (Fig. 1a; blue area: sensitive for ammonia emission; red area: very sensitive for ammonia emission) they were redefined as fuzzy object that represented a gradual transition of the protection zone (Fig. 1b), with additional planning rules to describe under which conditions a greenhouse development would or would not be allowed within the zone.

This is discussed in more detail in the following sections. 
Fig. 1 a Transition areas based on $250 \mathrm{~m}$ crisp buffer (Duindam 2006). b Transition areas based on fuzzy borders (Duindam 2006)

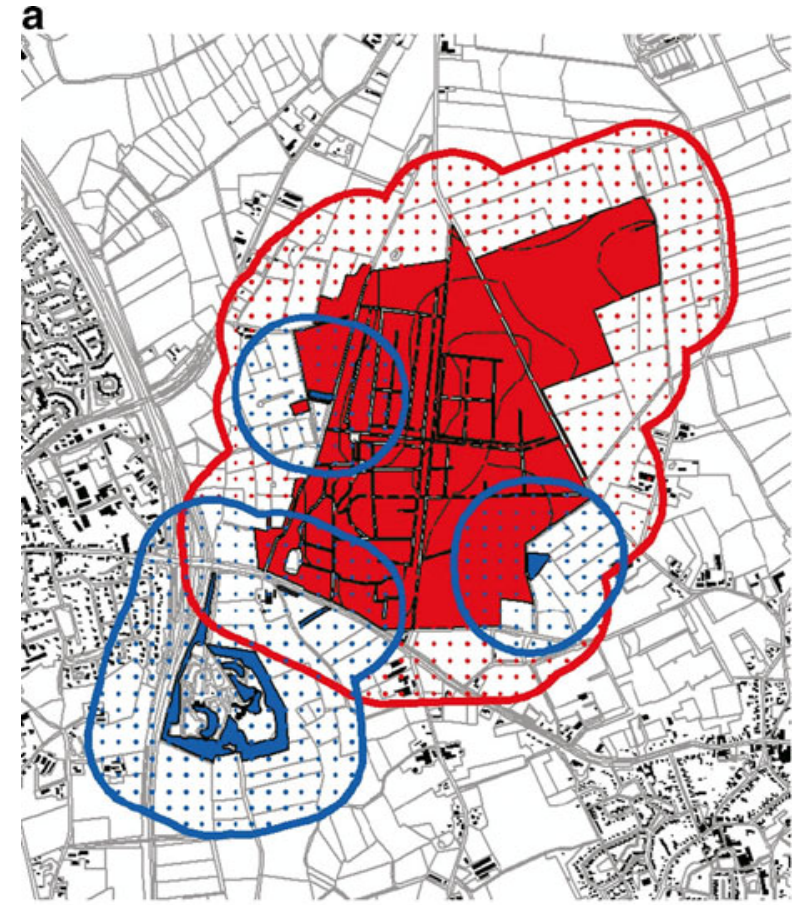

b

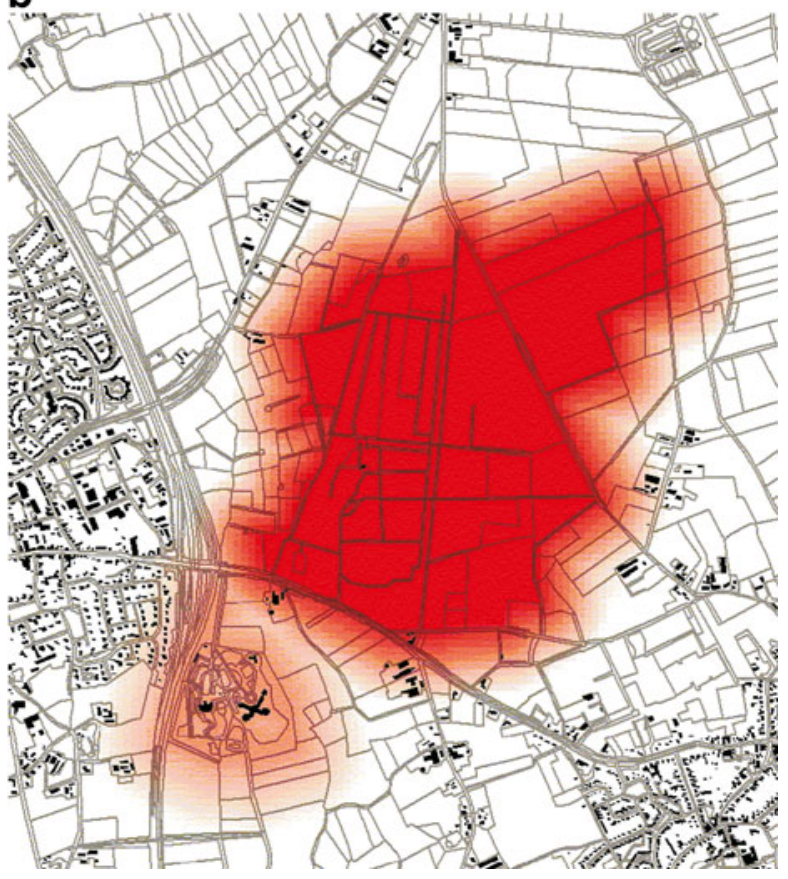


Several techniques can be used to represent continuous geographical phenomena in digital spatial plans. Bejaoui (2009) provided a broad overview of these techniques, starting with definitions based on exact representations. Examples include the eggyolk theory (Cohn and Gotts 1996), the definition of regions with broad boundaries (Clementini and Di Felice 1997) based on the principles of the general point-set theory and the theory of Erwig and Schneider (1997), where a region with a vague shape has a composed geometry. Furthermore, Bejaoui (2009), Shi (2010) and Comber et al. (2005) described other representations based on the probability, fuzzy set and rough set theories. Shi (2010) and Comber et al. (2005) concluded that the fuzzy set theory is suitable for vague objects.

According to the definition used in this paper, the best way to deal with uncertainty is to provide more information about an object. Fuzzy set theory provides a good approach to describe the continuous objects in a more realistic way and to reduce at least part of the uncertainty by providing more accurate information about the objects.

The concept of fuzzy logic was introduced in 1965 by Zadeh (Zadeh 1965) as an extension to Boolean logic. The principle of Boolean logic is that something is either true (1) or false (0). This idea is abandoned in fuzzy logic because something can also be partly true and partly false. Therefore, a fuzzy set is a collection of objects that have a gradual partnership to a collection of classes: an object can be part of a class (1) or not (0), but it can also be partly a member of this class (every value between 0 and 1 ). The rate at which a fuzzy object participates in a class is called the membership of this object to the class. This membership is determined by the fuzzy membership function that defines the fuzzy object by the gradual transition within the border area of the object.

Fuzzy logic has previously been applied in spatial planning (Leung 1983). Zimmerman (1991) described the use of fuzzy logic within a decision making process at the theoretical level. Leung (1983) identified spatial planning as a field in which the use of fuzzy logic could be successful. Steinhardt (1998) stated that by applying fuzzy logic to a landscape assessment of larger areas, their actual heterogeneity is better represented. Kurtener and Badenko (2003) combined fuzzy logic, geographical information systems and multi-criteria analysis to support the allocation choice and land suitability assessment. They provided a practical implementation of an investment allocation problem to the worst parts of the land drainage systems located in the Saint-Petersburg suburbs.

Although Robinson (Robinson 2003) provided an overview of various membership functions that can be used in spatial planning, Duindam (2006) concluded that it is still preferable to determine a new membership function for representing fuzzy objects because of specific criteria related to the behaviour of planning objects. This resulted in a parameterised membership function, called the Planning Object Membership function (POM), which is shown below.

$$
\mu(\mathrm{x})= \begin{cases}1 & \text { for } \mathrm{x} \leq \alpha \\ 1-\frac{1}{2}\left(\frac{\mathrm{x}-\alpha}{\beta-\alpha}\right)^{2^{\rho}} & \text { for } \alpha<\mathrm{x} \leq \beta \\ \frac{1}{2}\left(\frac{\gamma-\mathrm{x}}{\gamma-\beta}\right)^{2^{\rho}} & \text { for } \beta \leq \mathrm{x}<\gamma \\ 0 & \text { for } \mathrm{x} \geq \gamma\end{cases}
$$


The variables in this function can be defined as follows:

$\alpha$ Distance at which an object belongs completely to a class (or at which the membership value is 1 )

$\beta$ Distance at which an object belongs for $50 \%$ to a class (or at which the membership value is 0.5 ).

$\gamma$ Distance at which the object no longer belongs to a class (or at which the membership value is 0 )

$\rho$ Determines the shape of the membership function. It can be concave, convex or linear.

Implementation and Results

The "POM demonstrator" was used to evaluate the fuzzy logic approach in handling the uncertainty in this case study. In this demonstrator, the POM function was implemented in an easy-to-use ArcGIS/ArcMap extension that allowed us to create, save, visualise and analyse fuzzy objects. Fuzzy protection zones were created by defining the values of the parameters of the POM function and applying them to crisply defined protection zones. (Figure 2; units of $\alpha, \gamma$, and $\beta$ are meters).

Furthermore, the POM-demonstrator has the functionality to add Planning Rules to the calculated membership values, or categories thereof. The POM demonstrator offers flexibility in terms of defining class boundaries, number of classes and planning rules describing the classes. For instance, these planning rules can be derived from existing spatial plans or can be based on the tacit knowledge of a group of experts about the spatial impact of light pollution and noise emission.

The planning rules add extra information to the fuzzy protection zones. During this case study we defined four classes to describe the conditions under which the allocation of a greenhouse is or is not allowed:

- Class 1: Greenhouses allowed $(0.1>\mu(\mathrm{x})>0)$;

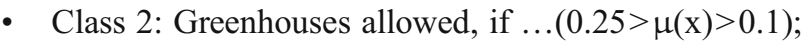

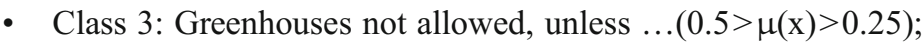

- Class 4: Greenhouses not allowed $(1>\mu(x)>0.5)$.

Fig. 2 Interactively defining a POM function with the POM demonstrator

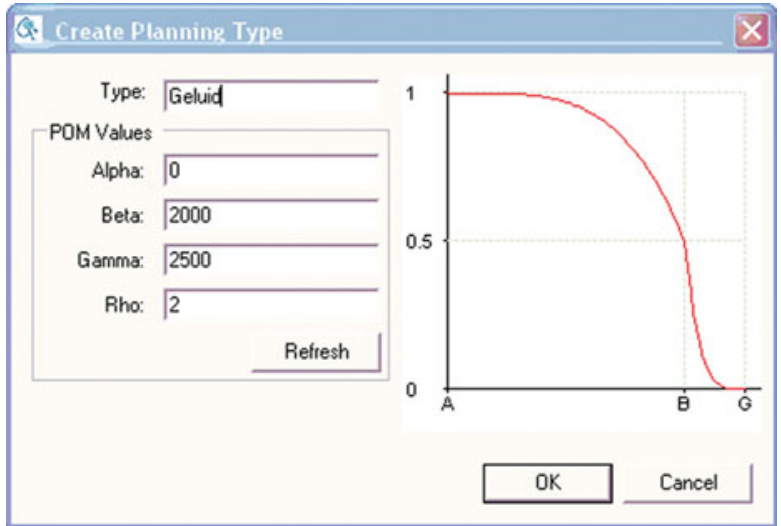


These conditions were set by spatial planning experts with knowledge of light pollution and noise emission. The fuzzy protection zones offered more flexibility for the allocation of greenhouses within the city triangle, under certain conditions even within the protection zones. The case study illustrated that the application of fuzzy logic to deal with uncertainty in the generation of digital plans at a regional planning level can provide options for the allocation of spatial planning objects that would otherwise be impossible.

Figure 3a shows the digital spatial plan generated with the conventional approach, where the greenhouses have been completely excluded from allocation in the $3 \mathrm{~km}$ buffer zones. In contrast, Fig. 3b shows the digital plan generated after applying the proposed fuzzy logic technique. Four classes are found as described below:

(1) Greenhouses allowed (transparent)

(2) Greenhouses allowed, if ...(Yellow)

(3) Greenhouses not allowed, unless ...(Orange)

(4) Greenhouses not allowed (Red)

Both classes 2 and 3 allowed us to allocate greenhouses in the region under certain conditions derived from contextual information generated by adding expert knowledge and policy measures using the POM function. As a result, a larger area is available for allocating greenhouses in the region, and it can be represented in a digital spatial plan.

\section{Discussion}

This case study showed that fuzzy logic offers more flexibility than crisp objects for dealing with the uncertainty of continuous phenomena such as noise emission in buffer zones surrounding Natura2000 sites. However the case study also showed that dealing with environmental factors in a quantitative way requires a different spatial planning approach. Setting the parameters of the POM function and classifying the membership values was not easy, not even for the experts. Normally, environmental factors are dealt with in a more intuitive way; valorising these relationships was new and very challenging. The advantage of using the POM demonstrator was that it immediately showed the implications of choices, which led to useful discussions and decisions about environmental relationships, such as noise.

However, the policymakers noted that introducing fuzzy objects in plans was impossible; by definition, legally bound plans cannot contain uncertainty. Although this is true, the new Dutch spatial planning system allows both legally bound plans (generally on the level of municipalities) and non-legally bound plans (at the local, regional and national levels). The proposed approach could provide solutions to the latter. Moreover, it should be noted that in our research project we did not want to restrict ourselves to the current law or other organisational agreements. This resulted in solutions that might not be legally feasible at present, but will offer ideas for changes in the future.

The participants in this case study (Dutch Ministry of Agriculture and the Dutch Cadastre) indicated that the method had potential. They were interested in the fuzzy logic approach and wanted to pursue further development. 
a

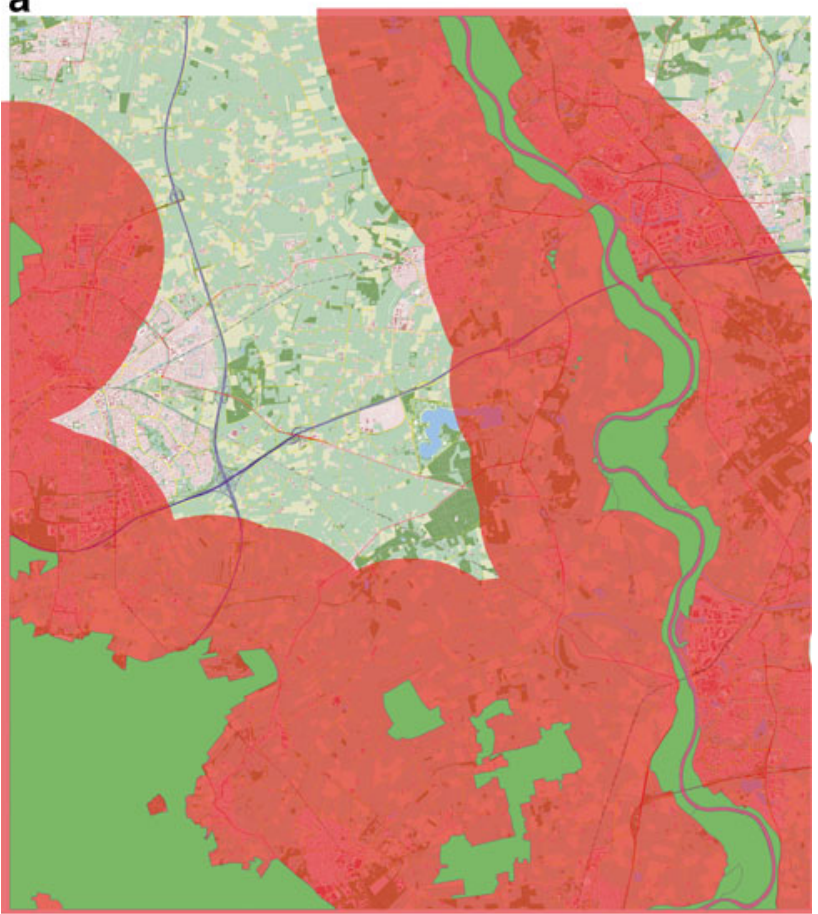

b

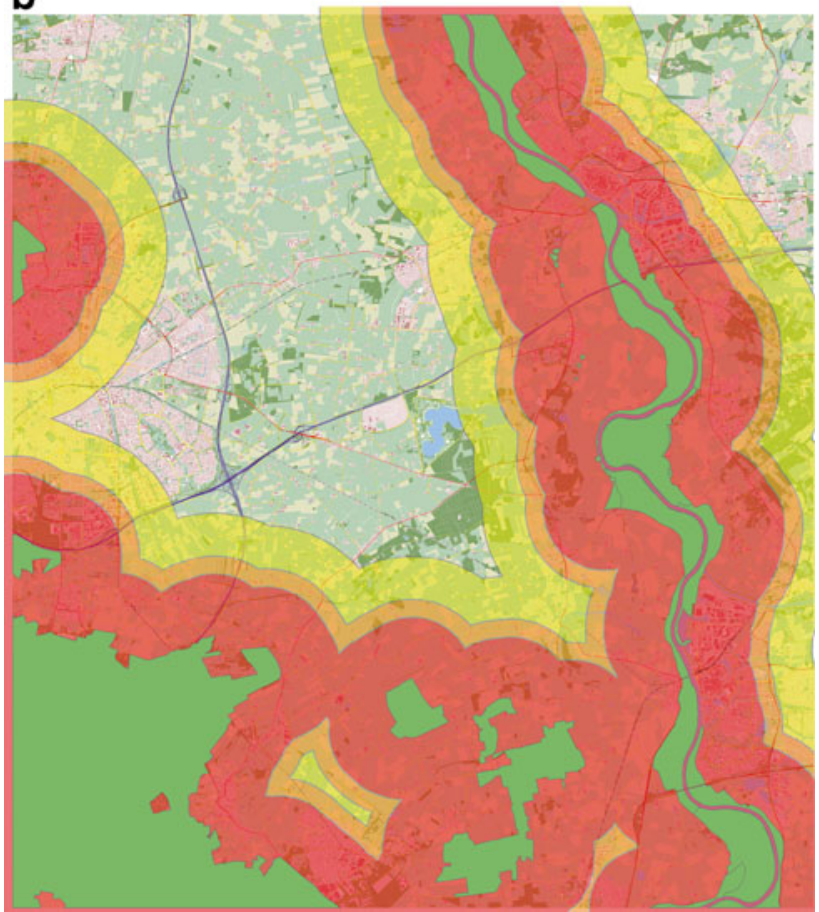

Fig. 3 a The area available as placement area with the conventional method. The natura2000 areas are green. b The area available as placement area with the fuzzy method 


\section{Case Study 2: 'Visualisation of Uncertainty in National Spatial Plans'}

The objective of this case study was to reduce interpretation problems due to uncertainties related to incomplete sources of information about planning objects through visualisation of those uncertainties in spatial planning maps. The main aim was to make users aware - from a planning perspective - of the intended and unintended uncertainties related to the planning objects. We focused on the visualisation of partially described objects found in maps at the national planning level. Although the selection of appropriate topographical base maps is also an issue (particularly if maps at different planning levels are combined), our research focused on the representation of the planning objects at various scale levels, and not on base maps.

\section{Background}

Maps have always been important in spatial planning, and as long as these maps have been issued as drawings or prints, the producers had full control over the content. Map use was mainly limited to the interpretation of the content. Digital spatial plans, however, have provided stakeholders more control over handling these maps: they can select and combine thematic layers or representations of objects from different sources and produce their own thematic maps that contain a variety of planning objects. This procedure may result in a combination of representations of planning objects extracted from thematic layers that provide incomplete, global indications of future land use. For example, the maps may contain a layer depicting local plans in detail. The combination of representations of planning objects that are extracted from different contexts and scales may lead to misinterpretations of a digital spatial plan.

Stakeholders should therefore be made aware of the context of use and be informed about aspects such as the aim (the 'message') of a digital spatial plan, its planning status (e.g. decreed or not, and by whom), the intended users and the user guidelines (e.g. the correct scale levels). This information can be provided through metadata, text windows in a map interface, pop-ups and hyperlinks to text documents. However, verbal information alone is not enough. This is because stakeholders are more confident in taking their decisions when uncertainties related to spatial planning objects are visualised on a digital spatial plan (Edwards and Nelson 2001). Leitner and Buttenfield (2000) demonstrated that adding information about uncertainty clarifies (rather than complicates) decision making at a national planning level.

Finally, Aerts et al. (2003) found that urban planners are aware of the usefulness of visualising uncertainty on digital spatial plans. Currently, digital spatial plans in the Netherlands often lack a graphic representation of uncertainty, or representations vary, making the information difficult to interpret and compare. This paper describes the first attempts to reduce interpretation problems in a digital environment and to increase awareness about uncertainty caused by partial descriptions of planning objects across spatial planning levels using a case study. 
The Uncertainty Visualisation Approach

The steps of the approach to the problem are illustrated in Fig. 4. For the first stepanalysis of the nature of uncertainty due to incompleteness in the maps - we selected six planning objects with different partial descriptions and characteristics:

1. Possible locations of 'greenports' and other horticultural centres that are currently represented by point symbols varying in shape or colour.

2. Search Areas for robust connections as part of the Ecological Main Structure that are currently represented by either arrows or lines attached to landscape elements such as rivers.

3. Search Areas, newly designated for nature conservation as part of the Ecological Main Structure, that are currently represented by coloured polygons with crisp outlines.

4. Possible locations of protected areas designated as National Landscapes that are currently also represented by coloured polygons and crisp outlines.

5. Locations of sources of noise or odour nuisance currently represented using point symbols within an affected area, which is usually represented as having a crisp boundary.

6. Locations of sources of noise or odour nuisance of which the affected areas vary temporally (e.g. with prevailing wind directions, with daily and weekly traffic patterns) and which are currently not represented on a digital spatial plan.

Analysis of the nature of uncertainty due to incompleteness of these planning objects revealed that the uncertainties are related to spatial accuracy; this can be further classified into uncertainties in spatial boundaries and uncertainties in the location of the entire planning object or parts thereof (Fig. 5).

The classification of types of uncertainty was followed by a literature review. The research work on the visualisation of uncertainty of geo-information has increased since the 1990s, when Beard et al. (1991) made an early attempt to propose a

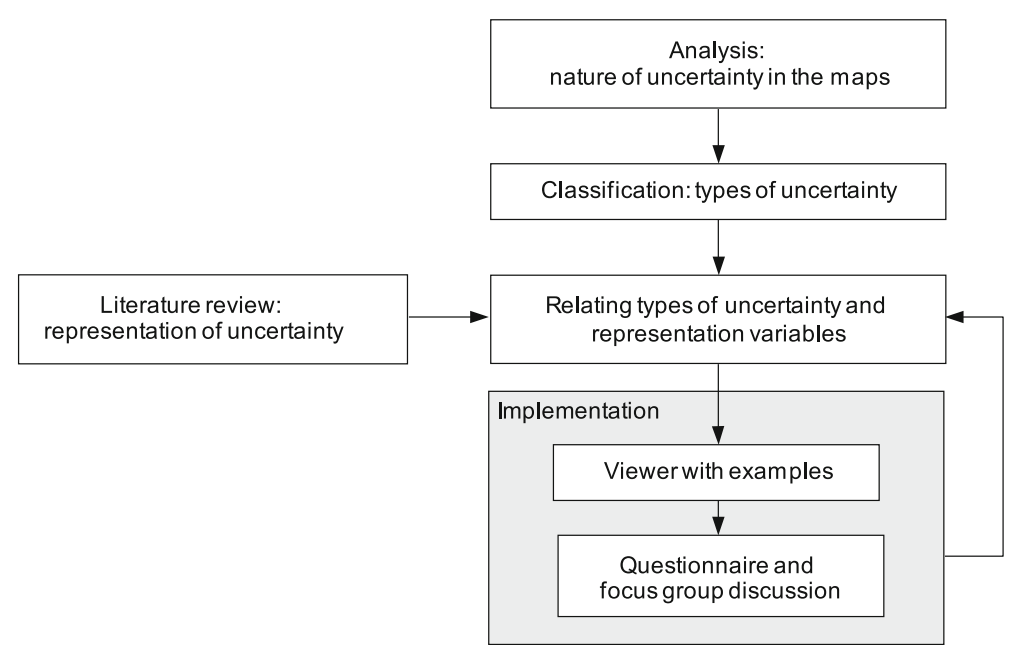

Fig. 4 Approach taken for the visualisation of uncertainty 


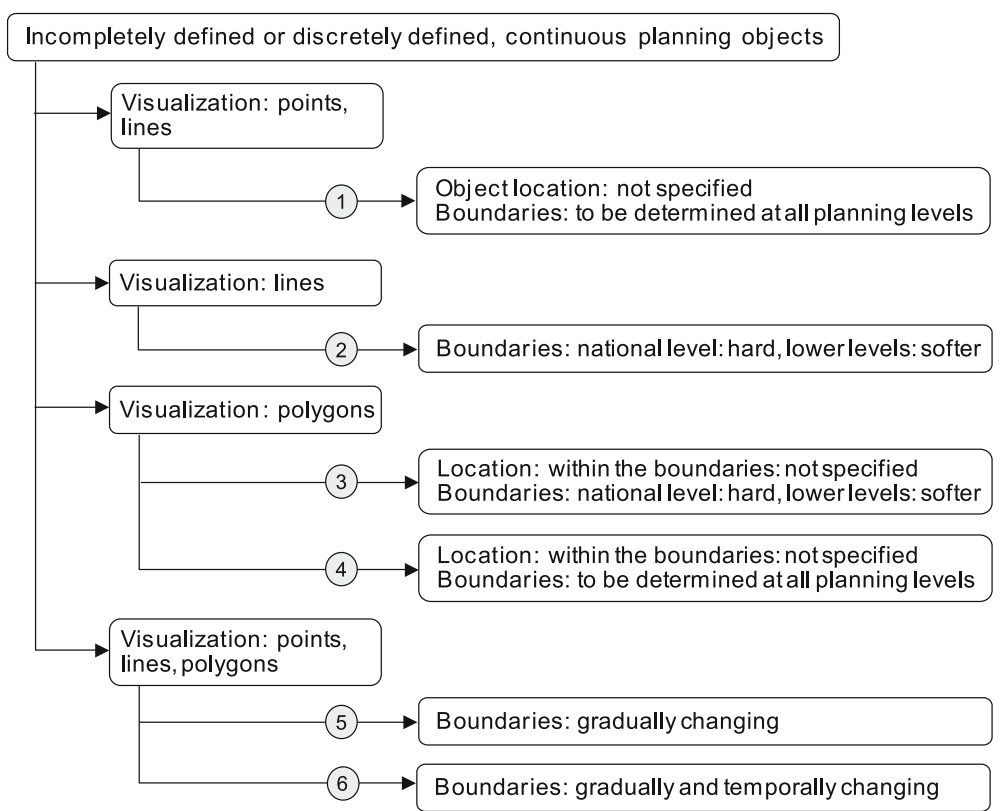

Fig. 5 Classification of types of uncertainty of planning objects. Numbers 1-6 refer to selected representatives of a range of planning objects with partial descriptions (see Approach section)

systematic framework for the cartographic representation of uncertainty. Recent overviews of uncertainty visualisation have been provided by MacEachren et al. (2005) and Slocum et al. (2009). MacEachren (1992) distinguished between three general methods of uncertainty visualisation: 1 . visualising data characteristics and uncertainty in two separate maps, 2 . visualising data characteristics and uncertainty in a single map, 3. making an interface that enables users to manipulate (e.g. change parameters) and control the visualisation (e.g. toggle between data and uncertainty representation, or switch uncertainty off).

Gershon (1998) explained that uncertainty visualisation needs metaphors and cues to create intuitive representations of uncertainties, such as dashed instead of continuous lines, thick blurred or transparent instead of thin (crisp) lines, small arrows attached to points and lines, and schematic instead of realistic representations of objects. Many authors have indeed applied more or less intuitive methods that either reduce the visibility of uncertain objects (e.g. by making them darker, coarser, more transparent or fuzzier) or otherwise evoke an impression of an unclear context (e.g. with 'floating' symbols).

Overall, many uncertainty visualisations have been created using the well-known graphic variables originally proposed by Bertin (1974), mainly the ones that enable ordinal or higher perception: texture, value and size. Later extensions of the basic set of graphic variables have also been frequently applied, sometimes in combination with Bertin's variables.

Figure 6 illustrates several examples of extensions of Bertin's graphic variables. However, note that MacEachren's transparency (MacEachren 1995) used an intervening foggy layer that obscures (darkens) uncertain data, while in Fig. 6 more transparency or lighter shading would mean more uncertainty. 


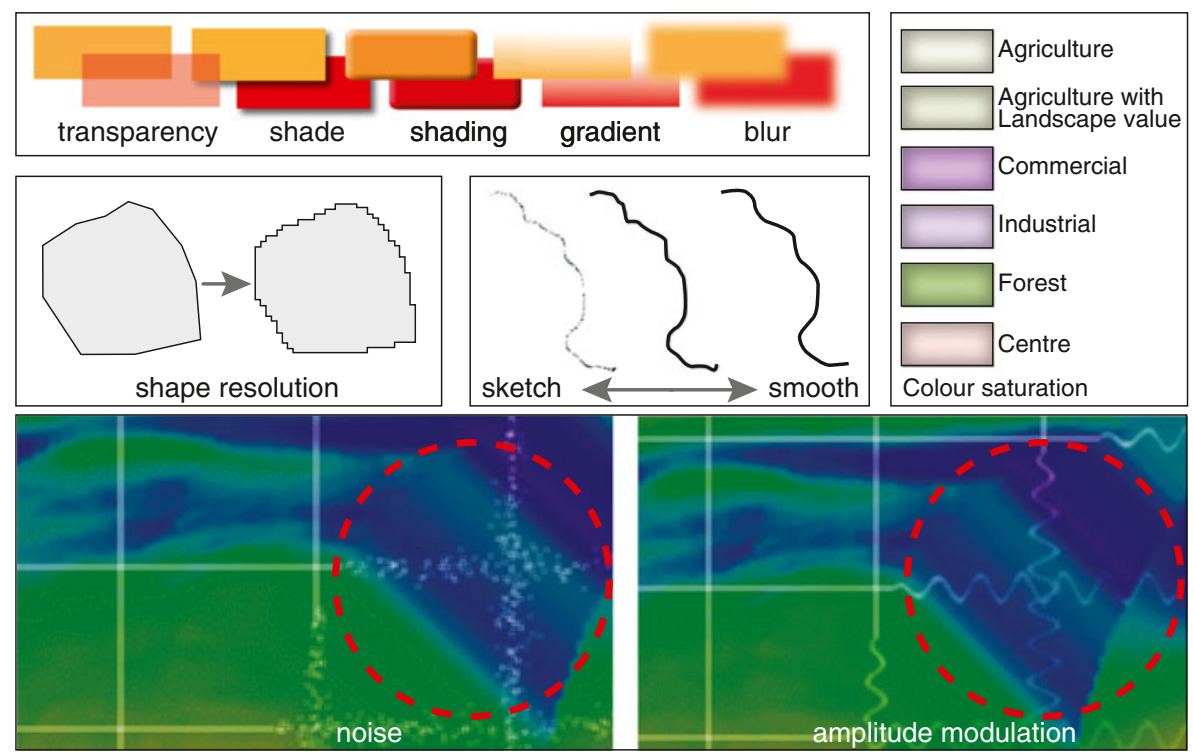

Fig. 6 Examples of extensions to the set of graphic representation variables defined by Bertin (1974). Noise and amplitude modulation: after Cedilnik and Rheingans 2000; C [2000] IEEE

Gradients (or continuous value change) have previously been used-among other techniques - to express fuzziness between class boundaries (Van der Wel et al. 1994). Shade and shading (Van den Worm 2001) enhance the contrast between figure and ground, and create an impression of floating symbols that are not sharply positioned. Saturation or relative purity of a colour (Morrison 1974) has been applied in many cases, although not always successfully. Success seems to depend on the context in which it is used: e.g.: whether uncertainty is represented in the same map as the data, or separately (Van Elzakker et al. 2007; Howard and MacEachren 1996); whether it is used in a static, dynamic or interactive map; and for which type of uncertainty (Van der Wel et al. 1994) it is used. Sketch lines or clearly smoothed lines are options to express low positional accuracy of planning objects. The graphic variables noise and amplitude modulation have been applied by Cedilnik and Rheingans (2000) to the lines of a grid overlaying a data visualisation. The grid represents data uncertainty, but the graphic variable noise can also be integrated in the visualisation of an object to express uncertainty.

Gershon (1998) suggested the use of small perpendicular arrows (or lines) may help to emphasise the uncertainty of a location of point or line symbols. Hatching (already commonly applied in digital spatial plans and equivalent to Gershon's dashed lines, but for areas or zones) may express unspecified locations within boundaries.

The above literature review led to a proposal to use some of the above mentioned graphic variables for the representation of the classified uncertainties related to planning objects in static visualisations (see Table 1 for the overview). Some of the proposed visualisations are graphically represented in Figs. 6, 7 and 8 (see below). However, a remark should still be made about planning objects that are often visualised by point or other abstract cartographic symbols. Although the exact boundaries of such planning objects may be uncertain, their intended size (e.g. in ha.) may be specified 
Table 1 Description of proposed visualisations; the graphic representation variables used for uncertainty are emphasised by italic text

\begin{tabular}{ll}
\hline Planning objects Static visualisation $\quad$ Dynamic visualisation \\
\hline
\end{tabular}

Possible locations of greenports and other horticultural centres

Search areas for robust connections as part of the Ecological Main Structure

Search areas newly designated for nature conservation as part of the Ecological Main Structure

Possible locations of protected areas designated as National Landscapes

Locations of sources of noise / odour nuisance
Fuzzy outlines of point symbols that are proportional in size to the planned area. Colour or form for types of centres.

If the locations of these planning objects are specified, and the boundaries are determined at national, but not at lower levels: crisp outlines on planning maps at national scales; on more detailed maps: increasing boundary uncertainties by increasing fuzziness (decreasing sharpness) or by increasing noise.

If neither the locations nor boundaries of these planning objects are specified: arrows with attached short perpendicular lines or arrows (for locational uncertainty). Alternative: small hatched zones, with increasing gradient in outward directions. Optional: colour for type of connection.

If boundaries are determined at national, but not at lower levels: crisp outlines on planning maps at national scales; on more detailed maps: increasing boundary uncertainties by increasing fuzziness (decreasing sharpness) or by increasing noise. Alternative: increasing coarser resolution of the shape of the whole area. Infill: gradient or hatching.

Increasing uncertainty about boundaries at lower levels: by increasing fuzziness (decreasing sharpness) or by increasing noise. Alternative: increasing coarser resolution of the shape of the whole area. Infill: gradient or moderately transparent hatching.

Gradient (continuous value) around the source, proportional in size to the average affected area. If different subzones can be distinguished: contours with different value infills (dark tints near a source, lighter tints at increasing distance from a source). Colour for type of source.

If representation of temporal uncertainty is also needed: transparent fog over the above mentioned symbols to reduce the visibility.
Instead of fuzzy outlines, slight repeated position changes (application of frequency) of the symbols. Colour (or form) and size as in the static visualisation.

Small, repeated position changes (application of frequency) of arrows without attached perpendicular elements or of hatched zones without gradient or transparency.

Slightly vibrating infill (application of frequency).

Slightly vibrating infill (application of frequency).

The transparent fog is replaced by repeated animated changes (application of frequency) in position or shape of the symbols.

in text documents. If that is the case, then the point or abstract cartographic symbol can be made proportional to the intended size of the planning object.

Blenkinsop et al. (2000) stated that during a spatial planning process, users generally prefer static maps, but animated uncertainty representations can provide an effective first impression of uncertainty. Therefore, we also proposed some dynamic visualisations in Table 1: slightly vibrating area infill, and small alternations in position or shape (Shepard 1994; Fisher 1996; see also Zhang et al. 2008; Fig. 7). These small repetitive changes are different realisations of the dynamic visualisation 

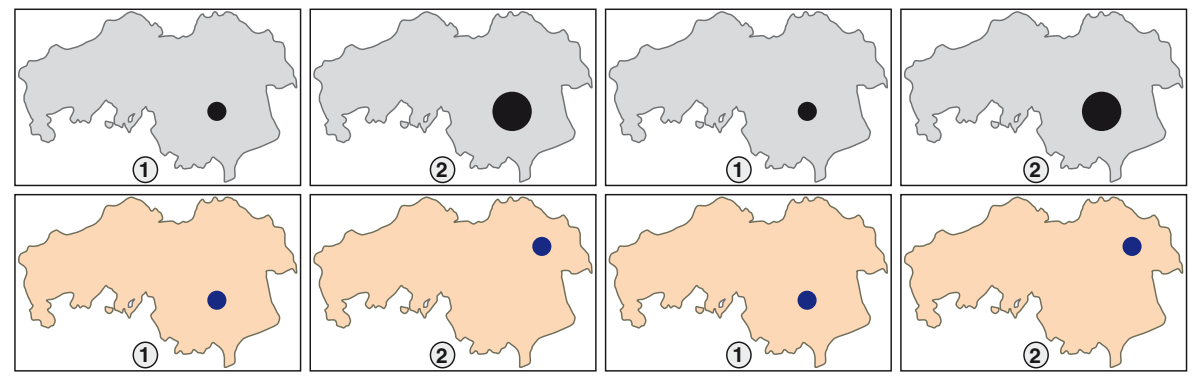

Fig. 7 Examples of dynamic visualisation of uncertainty, top: boundary uncertainty by repetitive display of a small (1) and a large (2) symbol ; bottom: location uncertainly by repetitive display of changes in location of a symbol $(1,2)$

of variable frequency (MacEachren 1994; Blok 2005) that attract attention, but may also distract the user from other aspects in the visualisation. Therefore, we also proposed that users should be able to switch the animated uncertainty visualisations on and off.
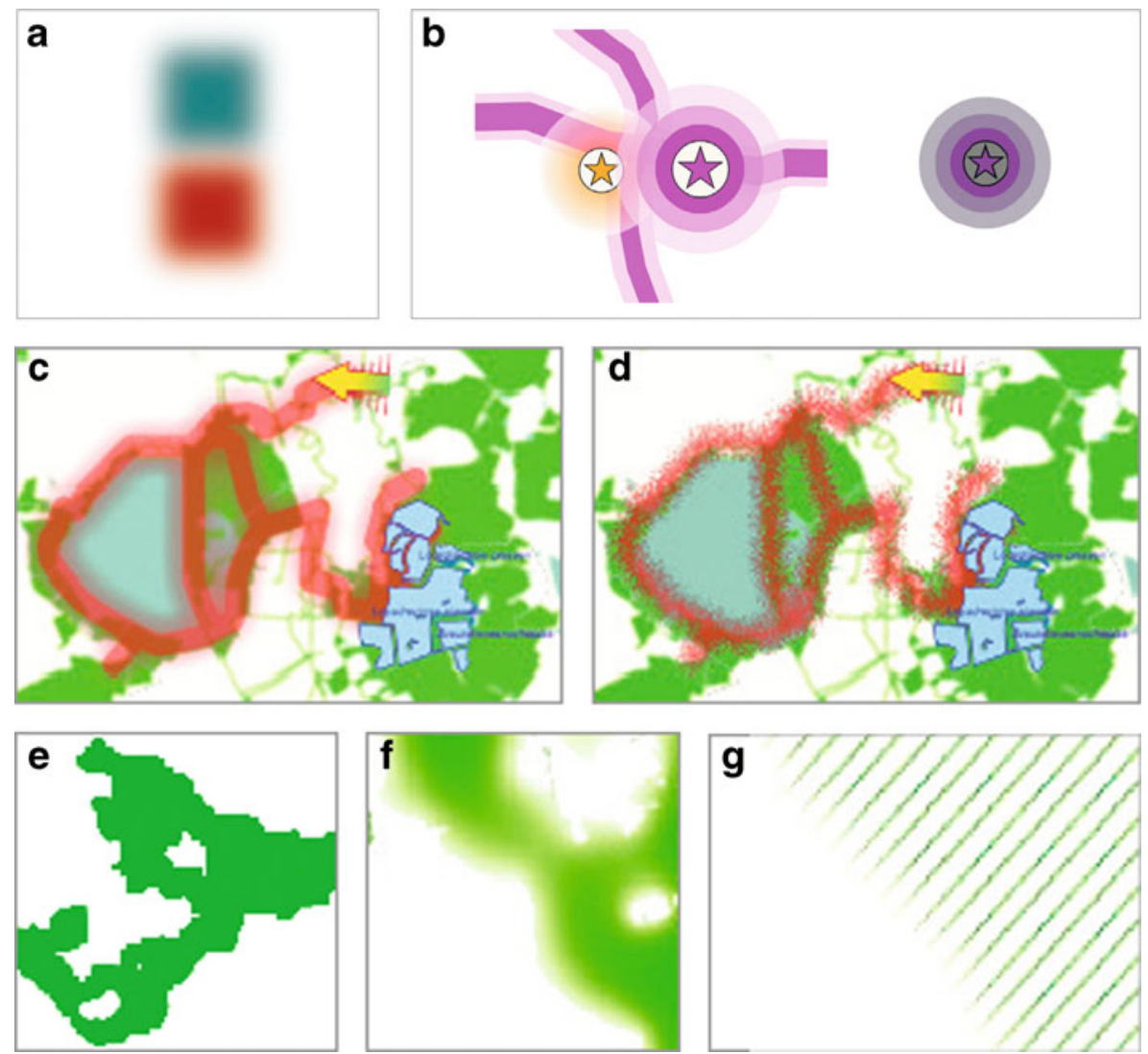

\section{g}

Fig. 8 Uncertainty visualisation: a. greenports and other horticultural centres; b. sources of noise or odour nuisance (without and with distinct zones; right: also being temporal uncertain); $\mathbf{c}$ and $\mathbf{d}$. robust connections by fuzzy lines noise; and by arrows if locations and boundaries are not yet defined; $\mathbf{e}$ and $\mathbf{f}$. polygon outlines (e.g. for designated areas) with shape resolution and fuzziness; e.g. polygon infill (hatching and gradient) 


\section{Implementation}

In 2006 the Dutch Ministry of Agriculture, Nature and Food Quality published their long-range plan for rural areas in a digital form, but they did not want to publish the maps that belonged to the plan on a regional and local scale in a viewer. This was because they were concerned about wrong interpretations by users who might, for example, overlay selections on detailed maps. Therefore, this was a good case to test improved visualisation as a proposed solution.

To demonstrate the changes in visualisation from the national to local level, a simple viewer was built, but due to time constraints we could not include all proposed visualisations. All graphic variables of the examples in Fig. 8 were available in the viewer, except the shape resolution, the area infill by hatching and by gradient. The examples of symbols for sources of noise or odour nuisance were useful in cases where areas were presented as distinct zones instead of continuous spatial objects. In other cases, gradients (as in the orange symbol) were better.

The visualisations were demonstrated in a workshop attended by producers and professional users of Dutch spatial planning maps. The participants $(n=14)$ filled in a questionnaire and took part in a focus group discussion. The aim of the session was to collect feedback and opinions about the visualisation principles applied. The visualisations were therefore not presented as indisputable solutions.

\section{Results and Discussion}

Like Aerts et al. (2003), we found that the participants of the workshop attached great value to the graphical representation of uncertainty in spatial planning maps. In general, the participants reacted positively to the proposed visualisation principles, which were based on literature review. Sometimes it was difficult to assess the different options, since the viewer had some limitations and not all variants were included (see Section 4.3). In spite of this, we obtained valuable feedback, which is summarised below.

Graphically fuzzy (rather than crisp) lines and boundaries were received most positively, although adaptations and further studies (e.g. in the width of the fuzzy lines in different use contexts) are needed. For robust connections, fuzzy lines were preferred over application of the graphic variable noise to the lines. Noisy lines were found to be too scrambled in the viewer, but nevertheless, noise was seen as a promising graphic variable, worth further investigation. According to the workshop participants, the artefacts attached to the arrows to emphasise location uncertainty could be removed; arrows on small scale maps were already conceived as relatively unfixed. The use of drop-shadows (shade; Fig. 9) might further strengthen the unfixed impression.

It became clear during the workshop that the outer boundaries of search areas for new nature within the Ecological Main Structure are not only fixed at the national
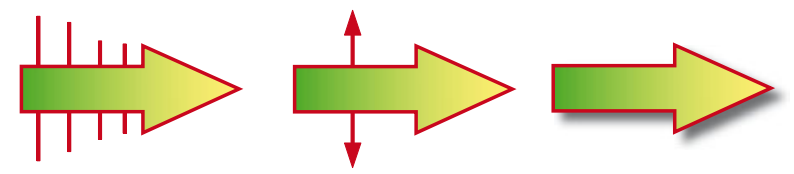

Fig. 9 Arrows for robust connections. Left to right: as realised in the viewer, preferred option before the workshop, and recommended after the workshop 
level, but also at all spatial planning levels. Within the fixed boundaries, lower tiers of government should decide where to realise their targets for new nature. Hence, the visualisation should be adapted: boundaries of these search areas should be represented as crisp polygons at all levels, and infills should reflect the uncertainty of location within the boundaries (e.g. by combining hatching and gradient). It also means that the boundary uncertainty of subgroup 3 in the classification presented in Fig. 5 should be removed.

For sources of noise or odour nuisance, the participants of the workshop preferred different visualisations at national and regional scales. Representation at the national scale could be limited to dots to show spatial distribution; details about affected areas are only needed at lower levels. Our idea to include, if relevant, the currently missing temporal uncertainty in the visualisation was received positively by $36 \%$ of the participants. But showing this by adding fog was unclear, so additional explanation was required, e.g. via mouse-overs.

Based on the results of the workshop, the proposed visualisations in Table 1 could be improved, as suggested in Table 2. In addition to the graphic variable noise, shape resolution (although not in the viewer) has been retained in the new table, since it seemed suitable for representing national landscapes, which have unfixed, broad boundaries.

Results obtained from the questionnaire and focus group discussion held during the workshop with stakeholders indicated that the proposed visualisation principles, although generally received positively, need further elaboration. User preferences, problems and suggestions resulted in an adapted proposal and ideas for future research on aspects like the width of fuzzy lines, use of gradients and noise as a graphic variable.

\section{Conclusions}

In conclusion, both case studies have shown a way of dealing with uncertainty caused by incomplete data. The question is whether these methods help to reduce the lack of confidence among planning officials in planning procedures, which was caused by recent changes in spatial planning legislation in the Netherlands. Both methods provide extra information to planning officials about planning objects. The fuzzy set theory provides extra information by converting the discrete borders of continuous objects into fuzzy borders that improve the resemblance to the real object and thus make it more realistic. As shown in the second case study, visualisation also improves the degree of realism and thus provides additional information. Both case studies showed that providing additional information reduces the uncertainty felt by planning officials.

The two solutions for dealing with uncertainty that are described in this paper received positive reactions in the case studies, but further research and development is needed for a full proof-of-concept and before the solutions can be applied generally. A true fuzzy logic approach requires a closer look at the translation of spatial planning phenomena into fuzzy logic functions. If successfully applied and properly transposed into legislation, this could enable a breakthrough in the societal acceptance of complex spatial planning matters. Furthermore, The POM demonstrator needs be fitted more closely to the planning process.

As a means to deal with uncertainty, visualisations are of great value, but they cannot stand on their own; they should be supplemented with uncertainty information in meta 
Table 2 Adapted description of proposed visualisations; graphic representation variables for uncertainty are again emphasised by italic text

\begin{tabular}{ll}
\hline Planning objects $\quad$ Static visualisation $\quad$ Dynamic visualisation \\
\hline
\end{tabular}

Possible locations of greenports and other horticultural centres

Search areas for robust connections as part of the Ecological Main Structure

Search areas newly designated for nature conservation as part of the Ecological Main Structure

Possible locations of protected areas designated as National Landscapes

Locations of sources of noise / odour nuisance
Fuzzy outlines of point symbols that are proportional in size to the planned area. Alternative: mouse-overs for size of the planned areas. Colour or form for types of centres.

If locations and boundaries of these planning objects are determined: crisp outlines. If the boundaries still have to be determined, on more detailed maps: increasing boundary uncertainties by increasing fuzziness (decreasing sharpness) or by increasing noise.

If neither locations nor boundaries of these planning objects are specified: arrows with shadow to emphasise the uncertainties. Alternative: small hatched zones, with increasing gradient in outward directions. Optional: colour for type of connection.

Crisp lines for boundaries at all levels. Infill: gradient or hatching.

Increasing uncertainty about boundaries at lower levels: by increasing fuzziness (decreasing sharpness) or by increasing noise. Alternative: increasing coarser resolution of the shape of the whole area. Infill: gradient or moderately transparent hatching.

On national scale maps; only dots for the distribution of sources. On more detailed maps: gradient (continuous value) around the source, proportional in size to the average affected area. If different subzones can be distinguished: contours with different value infills (dark tints near a source, lighter tints at increasing distance from a source). Colour for type of source.
Instead of fuzzy outlines, slight repeated position changes (application of frequency) of the symbols. Colour (or form) and size as in the static visualisation.

Small, repeated position changes (application of frequency) of arrows without shadow or of hatched zones without gradient or transparency.

Slightly vibrating infill (application of frequency).

Slightly vibrating infill (application of frequency).

If representation of temporal uncertainty is also needed: repeated animated changes (application of frequency) in position or shape of the symbols.

data, linked documents, etc. This is particularly important in a use context where spatial planning objects have legal status. Given the growing importance of e-governance, a full proof-of-concept of the proposed visualisation methods requires additional research that involves citizens as well as professional users. This should lead to the specification and formalisation of visualisations in an interoperable form. Moreover, off-the-shelf GISs are not yet technically adapted to uncertainty visualisation (Hunter et al. 2009). All of the above steps will 
ultimately be needed to reduce interpretation problems related to the use of spatial planning maps.

Open Access This article is distributed under the terms of the Creative Commons Attribution License which permits any use, distribution, and reproduction in any medium, provided the original author(s) and the source are credited.

\section{References}

Aerts, J. C. J. H., Clarke, K. C., \& Keuper, A. D. (2003). Testing popular visualization techniques for representing model uncertainty. Cartography and Geographic Information Science, 30(3), 249-261.

Atkinson, P. M., \& Foody, G. M. (2002). Uncertainty in remote sensing and GIS: fundamentals. In G. M. Foody \& P. M. Atkinson (Eds.), Uncertainty in remote sensing and GIS (pp. 287-302). New York: John Wiley and Sons.

Beal M., Ghahramani Z. (2003) The variational Bayesian EM algorithms for incomplete data: with application to scoring graphical model structures. In: J. Bernardo, M. Bayarri, J. Berger \& A. Dawid (Eds.), Bayesian Statistics 7. Cambridge University Press.

Beard, M. K., Buttenfield, B. P., \& Clapham, S. B. (1991). NCGIA Research Initiative 7: Visualization of spatial data quality. NCGIA Technical Paper 91-26, Santa Barbara, CA.: National Center for Geographic Information and Analysis.

Bejaoui, L. (2009). Qualitative topological relationships for objects with possibly vague shapes: implications on the specification of topological integrity constraints in transactional spatial databases and in spatial data warehouses. Thesis (PhD). Faculte de foresterie et de geomatique, Universite Laval, Quebec, Canada.

Bertin, J. (1974). Graphische Semiologie, Diagramme, Netze, Karte. Originally published in French as: Sémiologie graphique (1967). Berlin: Walter de Gruyter.

Blenkinsop, S., Fisher, P., Bastin, L., \& Wood, J. (2000). Evaluating the perception of uncertainty in alternative visualization strategies. Cartographica: The International Journal for Geographic Information and Geovisualization, 37(1), 1-14.

Blok, C. A. ( 2005). Dynamic visualization variables in animation to support monitoring of spatial phenomena. Thesis (PhD). Nederlandse Geografische Studies/Netherlands Geographical Studies 328/ITC Dissertation 328. Utrecht/Enschede, Universiteit Utrecht/ITC: 188.

Cedilnik, A., \& Rheingans, P. (2000). Procedural annotation of uncertain information. Proceedings of IEEE Visualization, 2000(77-84), 542.

Clementini, E., \& Di Felice, P. (1997). Approximate topological relations. International Journal of Approximate Reasoning, 16, 173-204.

Cohn, A.G. and Gotts, N.M. (1996). The 'egg-yolk' representation of regions with indeterminate boundaries. In P. Burrough \& A. Frank (Eds.), Proceedingsof the GISDATA Specialist Meeting on Spatial Objects with Undetermined Boundaries, Taylor \& Francis, pp. 171-187.

Comber, A., Wadsworth, R., Fisher, P. (2005). Reasoning Methods for Handling Uncertain Information in Land Cover Mapping. In R. Devillers \& R. Jeansoulin (Eds.), Fundamentals of Spatial Data Quality. ISTE, London, 123-141.

Duindam, A. J. (2006). Fuzziness in spatial planning data - an exploration in uncertainty. Centre for geoinformation, WUR. Research report GIRS-2006-20.

Edwards, L. D., \& Nelson, E. S. (2001). Visualizing data certainty: a case study using graduated circle maps. Cartographic Perspectives, 38, 19-36.

van Elzakker, C. P. J. M., Feringa, W. F., van den Worm, J., Poppe, E. L., \& Köbben, B. J. (2007). Vergelijkbare bestemmingsplannen: advies cartografische verbeelding digitale bestemmingsplannen. Eindrapport opdracht Minister van VROM (p. 76). Enschede: ITC.

Erwig, M., \& Schneider, M. (1997). Vague Regions. In 5th International Symposium on Advances in Spatial Databases (SSD’97), Lecture Notes in Computer Science, 1262, pp. 298-320.

Fisher, P. F. (1996). Animation of reliability in computer-generated dots maps and elevation models. Cartography and Geographic Information Systems, 23(4), 196-205.

Fisher, P. F. (1999). Models of Uncertainty in Spatial Data. In P. Longley, M. Goodchild, D. Maguire \& D. Rhind (Eds.), Geographical information systems: principles, techniques, management and applications (vol. 1, pp. 191-205). New York: Wiley and Sons. 
Fisher, P. F. (2003). Data quality and uncertainty: Ships passing in the night! In W. Shi, M. F. Goodchild, \& P. Fisher (Eds.), Proceedings of the Second International Symposium on Spatial Data Quality (pp. 17-22). Hong Kong: Hong Kong Polytechnic University.

Gao, J. J., Chen, X. H., Li, J. Y., Liu, G. C., \& Ma, J. (2010). Irregular seismic data reconstruction based on exponential threshold model of POCS method. Applied Geophysics, 7(3), 229-238.

Gershon, N. D. (1998). Visualization of an imperfect world. Computer Graphics and Applications, 18(4), $43-45$.

Howard, D., \& MacEachren, A. M. (1996). Interface design for Geographic Visualization: tools for representing reliability. Cartography and Geographic Information Systems, 23(2), 59-77.

Hunter, G. J., Bregt, A. K., Heuvelink, G. B. M., de Bruin, S., \& Virrantaus, K. (2009). Spatial Data Quality: Problems and Prospects. In G. Navratil (Ed.), Research trends in geographic information science (pp. 101121). Springer-Verlag Berlin Heidelberg: Lecture Notes in Geoinformation and Cartography.

Kurtener, D., \& Badenko, V. (2003). Fuzzy Algoritms to support spatial planning. In: S. Geertman \& J. Stillwell (Eds.), Planning support systems in practice. Springer-Verlag.

Leyk, S., Boesch, R., \& Weibel, R. (2005). A conceptual framework for uncertainty investigation in map-based land cover change modelling. Transactions in GIS, 9(3), 291-322.

Leitner, M., \& Buttenfield, B. P. (2000). Guidelines for the display of attribute certainty. Cartography and Geographic Information Science, 27, 3-14.

Leung, Y. (1983). Fuzzy Sets Approach to Spatial Analysis and Planning, a Nontechnical Evaluation. In Geografiska Annaler. Series B, Human Geography, Vol. 65, No. 2, pp. 65-75.

MacEachren, A. M. (1992). Visualizing uncertain information. Cartographic Perspectives, 13, 10-19.

MacEachren, A. M. (1994). Time as a cartographic variable. In H. M. Hearnshaw \& D. J. Unwin (Eds.), Visualization in Geographical Information Systems (pp. 115-130). Chichester: John Wiley \& Sons.

MacEachren, A. M. (1995). How maps work Representation, visualization and design. New York: The Guilford Press.

MacEachren, A. M., Robinson, A., Hopper, S., Gardner, S., Murray, R., Gahegan, M., \& Hetzler, E. (2005). Visualizing geospatial information uncertainty: what we know and what we need to know. Cartography and Geographic Information Science, 32, 139-160.

Ministry of VROM. (2006a). De nieuwe Wet ruimtelijke ordening. Ruimte geven voor ontwikkeling. Stand van zaken 20 maart 2006. (www.vrom.nl).

Ministry of VROM. (2006b). Digitale plannen verplicht met nieuwe Wet ruimtelijke ordening. Stand van zaken mei 2006. (www.vrom.nl).

Ministry of VROM. (2010). Handreiking Interbestuurlijke uitwisseling van ruimtelijke plannen en besluiten-versie 1.1 .

Morrison, J. L. (1974). A theoretical framework for cartographic generalization with the emphasis on the process of symbolization. International Yearbook of Cartography, 14, 115-127.

Robinson, V. B. (2003). A perspective on the fundamentals of fuzzy sets and their use. In geographic information systems. Transactions in GIS, 7(1), 3-30.

Shepard, I. F. (1994). Symbols with attitude: time-varying symbolism and data visualization. Paper presented at the British Cartographic Society Annual Technical Symposium, University of Manchester.

Shi, W. (2010). Principles of Modeling Uncertainties in Spatial Data and Spatial Analyses. Boca Raton: Taylor and Francis Group.

Slocum, T. A., McMaster, R. B., \& Kessler, F. C. (2009). Thematic Cartography and Geovisualization. Upper Saddle River, NY, US: Pearson/Prentice Hall.

Steinhardt, U. (1998). Applying the fuzzy set theory for medium and small scale landscape assessment. Landscape and Urban Planning, 41(3-4), 203-208.

Vullings, L. A. E., Wessels, C. G. A. M., \& Bulens, J. D. (2009). Fuzziness to reduce uncertainty. In J. H. Haunert, B. Kieler, \& J. Milde (Eds.), Advances in GIS, 12th AGILE International Conference on Geographical Information Science. Germany: Hannover.

Vullings, L. A. E., \& de Vries, M. (2007). Dealing with uncertainty in spatial planning 10th AGILE International Conference on Geographic Information Science. Denmark: Aalborg University.

van der Wel, F. J. M., Hootsmans, R. M., \& Ormeling, F. J. (1994). Visualization of data quality. In A. M. MacEachren \& D. R. Fraser Taylor (Eds.), Visualization in modern cartography (pp. 313-331). Amsterdam: Elsevier.

Wessels, C. G. A. M., Vullings, L. A. E., \& Bulens, J. D. (2008). Omgaan met onzekere Planobjecten. Geo-Info, 2008-12, 464-469.

Williams, D. Liao, X., Xue, Y., Carin, L. (2005). Incomplete-data classification using logistic regression. http://citeseerx.ist.psu.edu/viewdoc/summary?doi=10.1.1.80.4794.

Worboys, M., \& Duckham, M. (2004). GIS: A computing perspective (2nd ed.). Boca Raton, FL: CRC Press, Inc. 
van den Worm, J. (2001). Web map design in practice. In M. J. Kraak \& A. Brown (Eds.), Web cartography: developments and prospects (pp. 87-107). London/New York: Taylor and Francis.

Zadeh, L. A. (1965). Fuzzy sets. Information and Control, 8(3), 338-353.

Zhang, J., \& Goodchild, M. F. (2002). Uncertainty in geographical information. London: Taylor and Francis.

Zhang, Q., Blok, C. A., \& Tang, X. (2008). Animated representations of uncertainty and fuzziness in Dutch spatial planning maps. Proceedings of the XXI ISPRS congress 2008. Volume XXXVII, Part B2, 10431048 .

Zimmerman, H. J. (1991). Fuzzy set theory- and its applications. The Netherlands: Springer. 